\title{
From Histology to Ultrastructure: The Use of Correlative Electron Microscopy in High School Biology Lessons
}

\author{
C. Queenan*, A. Calabro*, D. Becker*, R. Pergolizzi** \\ * Bergen County Academies, Nano-Structural Imaging Lab, 200 Hackensack Avenue, Hackensack, \\ NJ 07601 \\ ** Bergen County Academies, Stem Cell Lab, 200 Hackensack Avenue, Hackensack, NJ 07601
}

High schools and college biology labs have used animal dissection and histology as part of their curriculum for many years. Through these lessons, students are expected to learn proper lab techniques, apply the knowledge gained from lecture and textbooks and identify tissues and macrostructures using their eyes or basic light microscopes. While this is an important educational experience for students, technology can be used to improve upon these lessons and provide students with a more in-depth examination of organs and tissues. The use of correlative microscopic analysis of the dissected tissues, cells and sub-cellular components will provide students with a more complete experience than basic textbook teaching or labs alone, while at the same time serving to spark a greater interest in the field of science.

Multiple Sprague-Dawley rat organs were provided to biology department faculty by researchers from the animal facility at Englewood Hospital in Englewood, NJ. The animals were sacrificed at the hospital's animal facility and the organs (heart, kidney, thigh muscle, liver and lung) immediately submerged in fixative ( $4 \%$ glutaraldehyde $/ 2 \%$ formaldehyde in $0.2 \mathrm{M}$ sodium cacodylate buffer, $\mathrm{pH}$ 7.4) provided by the Nano-Structural Imaging Lab. Once the samples were received, students enrolled in an upper level biology research course examined the visible structures on the organs. This was followed by anatomical discussion linking macro-scale observations of the organs to their role and function in the body.

After visual examination, the organs underwent preparation for scanning electron microscopy (SEM) and transmission electron microscopy (TEM). SEM samples were diced, post-fixed with $2 \%$ osmium tetroxide, dehydrated in a graded series of ethanol, critical point dried, mounted and coated with gold. Samples were imaged with an FEI Quanta 200 3D. TEM samples were diced, post-fixed with $2 \%$ osmium tetroxide, dehydrated in a graded series of acetone, embedded and cured in epoxy resin. $100 \mathrm{~nm}$ sections were collected onto 200 mesh copper grids and post-stained with $2 \%$ uranyl acetate for one hour and $0.5 \%$ lead citrate for 15 minutes. Samples were imaged with a JEOL JEM-2100. For light microscopy (LM) analysis of the tissue, $500 \mathrm{~nm}$ sections were cut from the TEM blocks with a glass knife, collected on glass slides, stained with $1 \%$ toluidine blue in $2 \%$ borate/distilled water, rinsed, cover slipped and imaged to show the difference in resolution that can be observed between light and electron microscopy.

The first exercise was to compare the structures of cardiac muscle from the heart (Figure 1) and skeletal muscle from the thigh (Figure 2) at the microscopic level. The students were given hands-on time with each microscope to locate structures and learn the imaging techniques and theory behind each microscope. They were also involved in identifying observed structures, such as sarcomeres, myofibrils, satellite cells, red blood cells, nerves, nuclei and mitochondria, using reference materials when necessary. 
Involving students in the process of observing the in-tact organs followed by identifying nano-scale structures from the same organs in real time on the SEM and TEM allowed them to become more invested in the lesson, hopefully improving their understanding of the curriculum material.

\section{References:}

[1] P. Cross, et al., Cell and Tissue Ultrastructure: A Functional Perspective. (1993).

[2] The authors would like to thank Dr. Herbert Dardick and his team from Englewood Hospital for providing the school with the tissue samples, and acknowledge the administration of the Bergen County Technical Schools for their continued support of the research program.
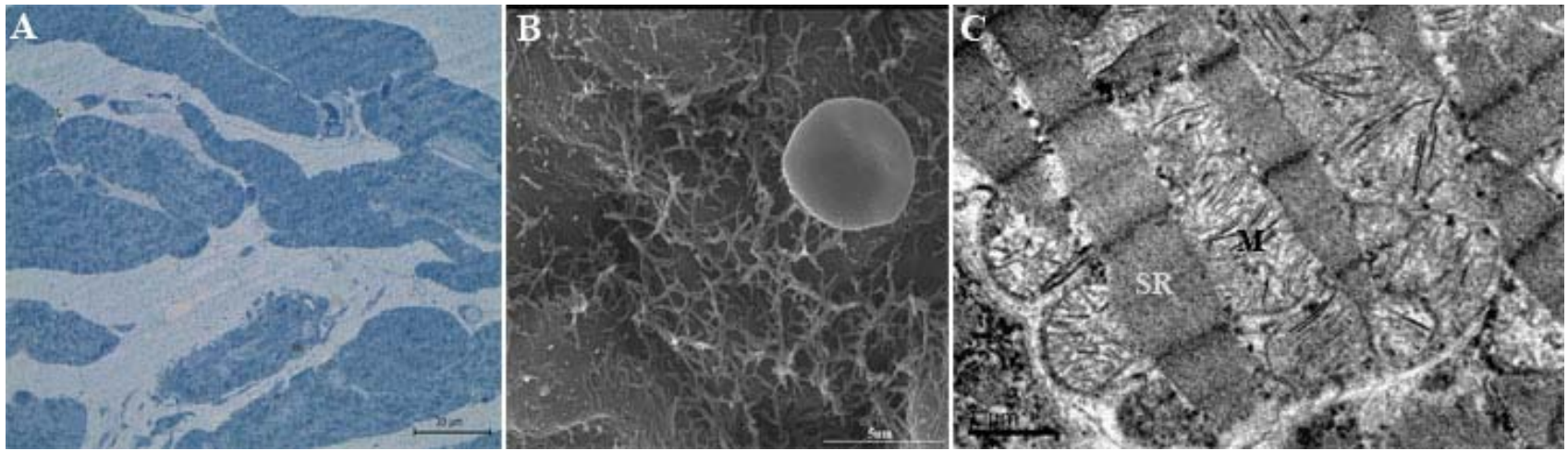

Figure 1: Images of rat heart. (A) LM image of cardiac muscle tissue showing branching. (B) SEM image of red blood cell and surface structure of the heart. (C) TEM image of cardiac muscle tissue. Labeled in the image are sarcomeres (SR) located in the myofibrils, as well as mitochondria (M) found between myofibrils. The number of mitochondria found within the muscle regions can relate to the oxidative contraction pattern of the cells [1].

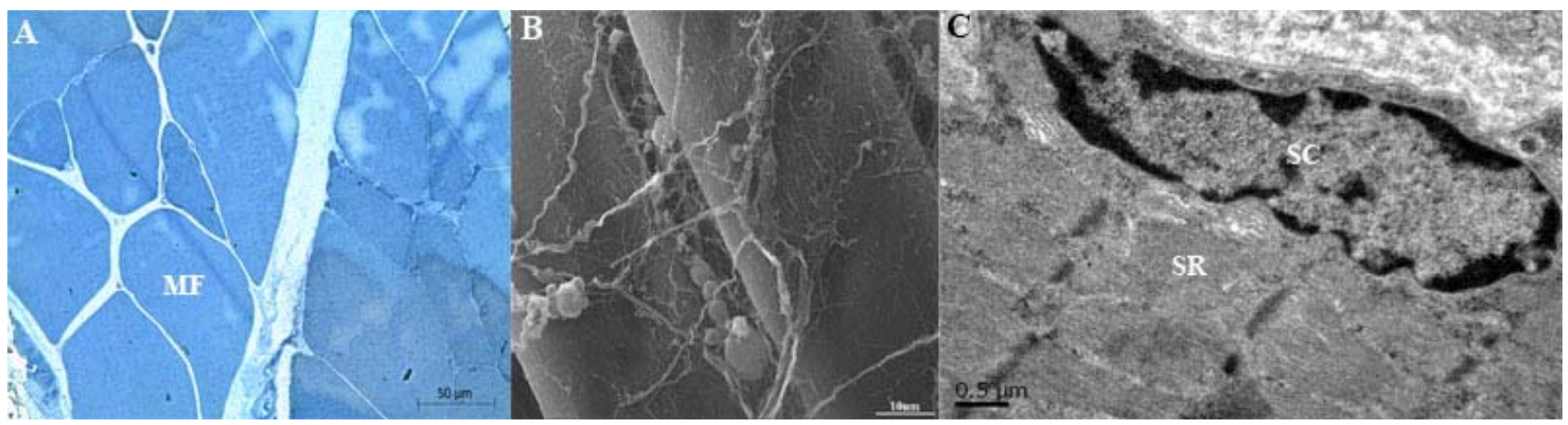

Figure 2: Images of rat thigh muscle. (A) LM image of the thigh's muscle fibers (MF) in cross section. (B) SEM image of a pair of muscle fibers and the nerve network spread along the surface of the fibers. (C) TEM image of a longitudinal section of the muscle fiber. Labeled in the image are sarcomeres (SR) located in the myofibrils, as well as a satellite cell (SC) which can be found approximately 1 for every 100 muscle fiber nuclei [1]. 